\title{
2kenize: Tying Subword Sequences for Chinese Script Conversion
}

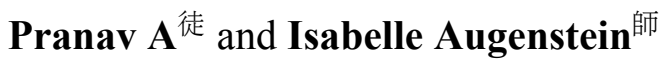 \\ 徒Independent Researcher, Hong Kong \\ 師Department of Computer Science, University of Copenhagen, Denmark \\ cs.pranav.a\{at\}gmail.com, augenstein\{at\}di.ku.dk
}

\begin{abstract}
Simplified Chinese to Traditional Chinese character conversion is a common preprocessing step in Chinese NLP. Despite this, current approaches have insufficient performance because they do not take into account that a simplified Chinese character can correspond to multiple traditional characters. Here, we propose a model that can disambiguate between mappings and convert between the two scripts. The model is based on subword segmentation, two language models, as well as a method for mapping between subword sequences. We further construct benchmark datasets for topic classification and script conversion. Our proposed method outperforms previous Chinese Character conversion approaches by 6 points in accuracy. These results are further confirmed in a downstream application, where 2 kenize is used to convert pretraining dataset for topic classification. An error analysis reveals that our method's particular strengths are in dealing with code mixing and named entities. The code and dataset is available at https : //github.com/pranav-ust/2kenize
\end{abstract}

\section{Introduction}

Chinese character (or script) conversion is a common preprocessing step for Chinese NLP practitioners (Zhang, 2014; Shi et al., 2011). Traditional Chinese (TC) and Simplified Chinese (SC) are the two standardized character sets (or scripts) for written Chinese. TC is predominantly used in Taiwan, Hong Kong, and Macau, whereas SC is mainly adopted in mainland China and SC characters are simplified versions of TC characters in terms of strokes and parts. Therefore, Chinese NLP practitioners apply script converters ${ }^{1}$ to translate the

\footnotetext{
${ }^{1}$ Most of these tools like OpenCC, Mafan, Hanziconv are generally widely used within Chinese NLP community, which can be attested popularity of their Github repos.
}

\begin{tabular}{ll|c}
\hline SC Sentence & 维护发展中国家共同利益 & Comments \\
\hline Segmentation & 维 护发 展中 国家 共同 利益 & 护发: haircare \\
Conversion & 維護髮展中國家共同利益 & $\boldsymbol{x}$ Conversion \\
\hline Segmentation & 维护 发展 中 国家 共同 利益 & 发展: develop \\
Conversion & 維護發展中國家共同利益 & $\checkmark$ Conversion \\
\hline
\end{tabular}

Table 1: Example sentence with two different segmentations, and resulting different conversions. The sentence translates to Safeguarding the common interests of developing countries. This is a recurring example in this paper. Also refer $\S$ F.5.

dataset into their desired language. This is especially useful for TC NLP practitioners because TC is less widely used and under-resourced as compared to SC.

Converting from TC to SC is generally straightforward because there are one-to-one correspondences between most of the characters, so conversion can be performed using mapping tables (Denisowski, 2019; Chu et al., 2012). However, conversion from SC to TC is an arduous task as some SC characters can be mapped to more than one TC character depending on the context of the sentence. A detailed analysis by Halpern and Kerman (1999) shows that SC to TC conversion is a challenging and crucial problem, as $12 \%$ of SC characters have one-to-many mappings to TC characters. Our experiments show that current script converters achieve sentence accuracy results of 55$85 \%(\S 3)$.

Another issue is that varying tokenization would lead to different results as Chinese is an unsegmented language, see Table 1 for an example. Off-the-shelf script converters would translate 维 护发展中国家共同利益 into 維護髮展中國家 共同利益, ${ }^{2}$ whereas the correct conversion is 維

\footnotetext{
${ }^{2}$ Throughout this paper, we color code ambiguous SC characters with brown, ambiguous TC characters with violet, vernacular Cantonese characters with teal. By scripts, we refer as to character sets, and we interchangeably use them in this paper.
} 
護發展中國家共同利益. Here, the SC character 发 (hair, issue) has two TC mappings, 髮 (hair, issue) and 發 (hair, issue), depending on the context and tokenization; which shows that this task is non-trivial.

Despite this being an important task, there is a lack of benchmarks, ${ }^{3}$ which implies that this problem is understudied in NLP. In this study, we propose 2 kenize, a subword segmentation model which jointly considers Simplified Chinese and forecasting Traditional Chinese constructions. We achieve this by constructing a joint Simplified Chinese and Traditional Chinese language model based Viterbi tokenizer. Performing mapping disambiguation based on this tokenization method improves sentence accuracy by 6 points as compared to off-the-shelf converters and supervised models. Our qualitative error analysis reveals that our method's particular strengths are in dealing with code-mixing and named entities. Additionally, we address the issue of a lack of benchmark datasets by constructing datasets for script conversion and $\mathrm{TC}$ topic classification.

\section{2kenize: Joint Segmentation and Conversion}

We employ subword tokenization, as it addresses the issue of rare and unknown words (Mikolov et al., 2012) and has been shown advantageous for the language modelling of morphologicallyrich languages (Czapla et al., 2018; Mielke and Eisner, 2019). This achieves improvements in accuracy for neural machine translation (NMT) tasks and has now become a prevailing practice (Denkowski and Neubig, 2017). The most widelyutilized method is Byte Pair Encoding (BPE, Sennrich et al. (2016)), a compression algorithm that combines frequent sequences of characters, which results in rare strings being segmented into subwords. Unigram (Kudo, 2018) and BPE-Drop (Provilkov et al., 2019) use subword ambiguity as noise, as well as stochastically-corrupted BPE segmentation to make it less deterministic. For NMT tasks generally, subword segmentation is seen as a monolingual task and applied independently on source and target corpora. We hypothesize that translation tasks, and specifically conversion tasks, as investigated here, would have a bet-

\footnotetext{
${ }^{3}$ The ChineseNLP website states that script conversion benchmarks and experiments currently do not exist: https://chinesenlp.xyz/\#/docs/simplified_ traditional_Chinese conversion
}

ter performance if segmentation were performed jointly. Hence, in this section, we describe our proposed method 2 kenize, which jointly segments by taking the source and its approximate target sentences into account. This motivates the main idea of this paper: We propose 2 kenize which jointly considers the source sentence and its corresponding target conversions by doing lookaheads with mappings.

\subsection{Outline of the proposed approach}

Given the possible SC character sequence $\mathbf{s}=$ $s_{1} s_{2} \ldots s_{n}$ and TC character sequence $\mathbf{t}=$ $t_{1} t_{2} \ldots t_{n}$, we want to find the most likely $\mathbf{t}$, which is given by the Bayes decision rule as follows:

$$
\mathbf{t}=\underset{\mathbf{t}^{\prime} \in T^{*}}{\arg \max } p\left(\mathbf{s}, \mathbf{t}^{\prime}\right)
$$

where $T^{*}$ denotes the set of all strings over symbols $\left(t_{i}\right)$ in $T$ (Kleene star). We divide this problem into two parts: finding the mapping sequence (2) and finding the TC sequence from mappings (7).

We define a mapping, which is given by $m_{i}=$ $\left(\mathfrak{s}_{i}, \mathfrak{t}_{i}\right)=\left(s_{j: k}, \mathrm{t}_{j: k}\right)$. Here, $\mathrm{t}_{j: k}=\left\{t_{j: k}^{1} \ldots t_{j: k}^{n}\right\}$ is a set of TC characters that correspond to the $\mathrm{SC}$ character in the mapping. Thus, a mapping sequence can be defined as a concatenation of mappings, which is $\mathbf{m}=m_{1} m_{2} \ldots m_{l}$. Let $\mathcal{M}$ be the superset of all possible mapping sequences and $\mathcal{M}(\mathbf{s})$ be the all mapping sequences resulting from s. Then, the best possible mapping sequence is given by

$$
\mathbf{m}=\underset{\mathbf{m}^{\prime} \in \mathcal{M}(\mathbf{s})}{\arg \max } p\left(\mathbf{m}^{\prime}\right)
$$

Morever, $p(\mathbf{m})$ can be expanded as such:

$$
\begin{aligned}
p(\mathbf{m}) & =p\left(m_{1} m_{2} \ldots m_{l}\right) \\
& =p\left(\begin{array}{cccc}
\mathfrak{s}_{1} & \mathfrak{s}_{2} & \ldots & \mathfrak{s}_{l} \\
\mathfrak{t}_{1} & \mathfrak{t}_{2} & \ldots & \mathfrak{t}_{l}
\end{array}\right) \\
& \approx p\left(\mathfrak{s}_{1} \mathfrak{s}_{2} \ldots \mathfrak{s}_{l}\right)+p\left(\mathfrak{t}_{1} \mathfrak{t}_{2} \ldots \mathfrak{t}_{l}\right) \\
& =p_{L M}\left(\mathfrak{s}_{1: l}\right)+\sum_{t \in \prod_{i} \mathfrak{t}_{i}} p_{L M}\left(t_{1: l}\right)
\end{aligned}
$$

After expanding the mapping sequences (4), we take an approximation by estimating this as the sum of likelihoods of two sequences formed due to co-segmentations (5). The set of possible TC sequences is given by the Cartesian product of $\mathfrak{t}_{i}$. These likelihoods can then be estimated using language model (LM) probabilities as shown in (6).

$$
\mathbf{t}=\underset{\mathbf{t}^{\prime} \in \mathbf{m}_{\mathbf{t}}}{\arg \max } p\left(\mathbf{t}^{\prime}\right)
$$




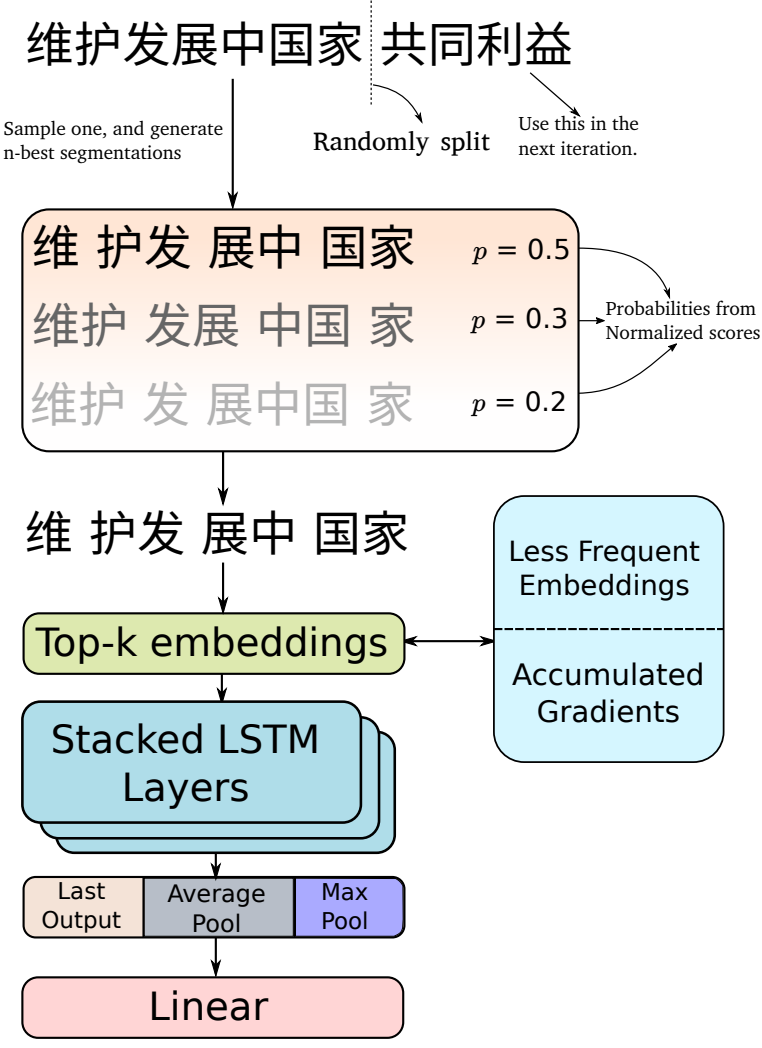

Figure 1: Language model architecture with subword and subsequence sampling. (Alt text: §F.1).

Once the mapping sequence $\mathbf{m}$ has been found, all possible TC sequences are found from the set $\mathbf{m}_{\mathbf{t}}$, which is the Cartesian product for all $\mathfrak{t}_{i}$ in $\mathbf{m}$. From (7), we calculate approximate final sequence using beam search.

\subsection{Model Architecture}

Viterbi, a dynamic programming (DP) algorithm, considers phrases (or subsequences) and performs segmentation in a 'bottom-up' fashion (Nagata, 1994; Sproat et al., 1996). RNN-based language models are theoretically considered to be ' $\infty$ 'gram (Khandelwal et al., 2018), which consitutes a challenge. Consider this sentence, 维护发展 中国家共同利益. A potential challenge could be to adquately estimate the probability of 共同 利益. As this sequence occurs infrequently in the beginning of sentences in the corpus, an RNN would under-estimate the probability of this subsequence. Moreover, an RNN would likely lose some useful context and perform worse without it (Kim et al., 2019). So for Viterbi to perform well with an RNN, we train the language model on subsequences. We approach this by training our model in such a way that it samples subsequences ran-

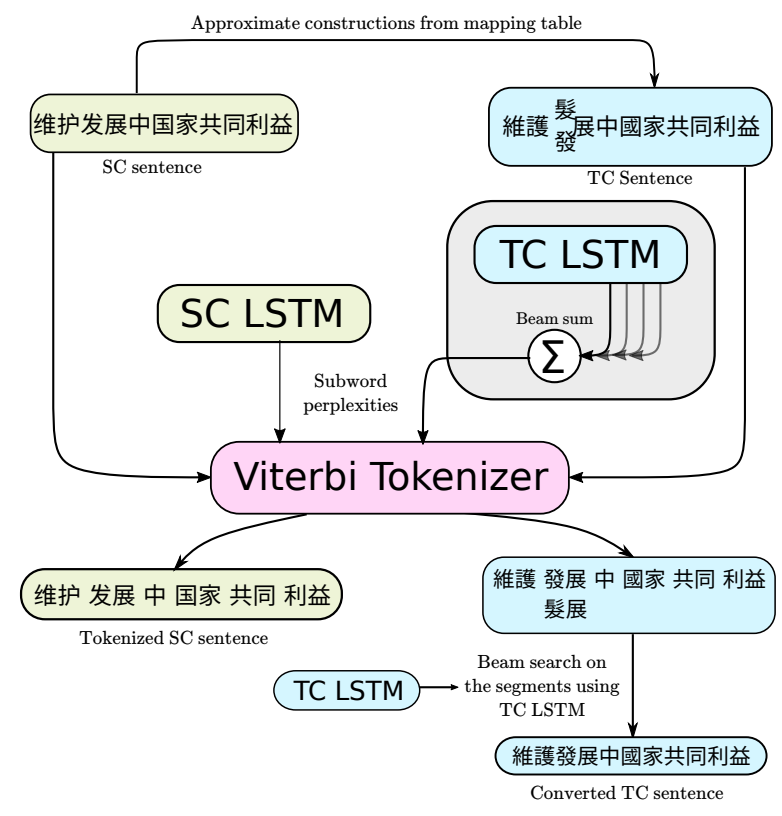

Figure 2: From the given SC sentence, we create possible TC sequences using mappings. We input these to Viterbi, which recursively calls LSTM. Using Eq. (6) as the scoring function, Viterbi outputs the mapping sequence. We perform beam search to find the best TC sequence from the mapping sequence. (Alt text: §F.2).

domly in each epoch. As shown in Fig 1, we randomly split the sentence and use subsequences in separate epochs.

Using Kudo (2018) regularization method, we sample from the $n$-best segmentations in each epoch. This is done so that the model can understand different segmentations of a subsequence using a similar motivation as above. Recent works have shown that varying subword segmentations lead to a better downstream model performance (Provilkov et al., 2019; Kudo, 2018; Hiraoka et al., 2019); therefore, we use it as a data augmentation strategy. Once we get the $n$-best segmentations with scores, we normalize them, and then use the normalized scores as sampling probabilities (see Fig 1). As opposed to other subword tokenizers where the vocabulary size is fixed, we do not limit the vocabulary in our model. Hence, there are numerous possibilities of segment combinations which raises a need of caching most frequent tokens. Inspired by the work related to cache-based LMs (Kawakami et al., 2017) and ghost batches (Hoffer et al., 2017), we only consider the top$k$ tokens in the main network memory and keep track of gradients of less recently used token embeddings (commonly known as LRU, Least Recently Used policy). This could be thought of as 


\begin{tabular}{|c|c|c|c|c|}
\hline & HK Literature & HK News & TW Literature & TW News \\
\hline \multirow[t]{3}{*}{ Sources } & Liu (1962) & Singpao (2017-2018) & Jiubadao (2011) & AS subset Emerson (2005) \\
\hline & Lau Yee (1972) & Mingpao (2017-2018) & Ko (2010) & Liberty Times (2017-2018) \\
\hline & Foon (1988) & CityU subset Emerson (2005) & Yao (1964) & United Daily News (2017-2018) \\
\hline Average Length & 194.8 & 214.6 & 188.2 & 223.6 \\
\hline$I A A$ & 0.982 & 0.979 & 0.981 & 0.971 \\
\hline Mapping Examples & 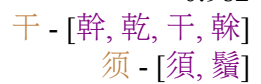 & $\begin{array}{c}\text { 苏 - [蘇, 蘇, 延] } \\
\text { 暗 - [暗, 闇] }\end{array}$ & $\begin{array}{c}\text { 复 - [復, 複, 覆] } \\
\text { 叹 - [嘆, 歎] }\end{array}$ & $\begin{array}{r}\text { 胡 - [胡, 博, 髶] } \\
\text { 迹 - [ 蹟, 跡 }]\end{array}$ \\
\hline
\end{tabular}

Table 2: An overview of the dataset used for intrinsic evaluation. We report sources, average character lengths and sentence level inter-annotator agreements (IAA, reported in $\kappa$ ) and some examples of ambiguous SC-TC mappings.

virtual embeddings as delayed gradient accumulation allows to accommodate larger number of tokens. This virtual size embedding architecture is related to the continuous cache implementation and stochastic tokenization architectures (Grave et al., 2016; Hiraoka et al., 2019).

\subsection{Segmentation and Disambiguation}

This optimal sequencing problem can be formulated as an overlapping subsequence approach, which can be solved using LM based Viterbi (Nagata, 1994; Sproat et al., 1996). Fig. 2 explains this process of joint subword modelling. Here, we take Eq. (6) as the objective function for finding the mapping sequence, however, we use subword perplexities (Cotterell et al., 2018; Mielke et al., 2019; Mielke, 2019) in our implementation. For the TC LSTM, we add the probabilities of the beams of the possible sequences.

As discussed in $\$ 2.1$ and Eq. (7), beam search is needed to select the best subword sequence for TC. Once the sentences are tokenized, the mapping table is used to convert each SC token to the corresponding TC token. We extract the final TC sentence by resolving ambiguities through beam search using the TC LSTM (Fig. 2).

\section{Intrinsic Evaluation}

\subsection{Dataset for Intrinsic Evaluation}

We construct a gold standard corpus for both Chinese scripts consisting of 4 domains: HK Literature and Newswire, and Taiwanese Literature and Newswire (Table 2) with each domain containing 3000 sentences. SC-TC mapping tables are constructed from existing resources (Denisowski, 2019; Chu et al., 2012). We heuristically convert selected TC sentences to SC using OpenCC. We asked the annotators to manually correct any incorrect conversions. ${ }^{4}$

\footnotetext{
${ }^{4}$ A detailed data statement is given in the appendix.
}

\subsection{Language Model Training}

We choose the SIGHAN-2005 Bakeoff dataset to train the segmentation-based language model (Emerson, 2005). For SC, we select the PKU and MSR partitions, and for TC, we use the Academia Sinica and CityU partitions. We apply maximal matching (or heuristic dictionary-based word segmenter) to pre-process these datasets by segmenting words into subwords (Wong and Chan, 1996). Here, 'dictionary' refers to the word-list in the mapping table. We then train a 2-layer LSTM language model LSTM with tied weights, and embedding and hidden sizes of 512 (Sundermeyer et al., 2012) on this segmented dataset with subsequence sampling and stochastic tokenization as discussed in $\S 2.2$.

\subsection{Baselines and Ablations ${ }^{5}$}

We implement the following baselines for the experimentation:

Off-the-shelf Converters: Hanziconv ${ }^{6}$ and $\mathrm{Mafan}^{7}$ are dictionary-based script character converters. Evaluating this could be useful to understand the lower accuracy bound. OpenCC ${ }^{8}$ uses a hybrid of characters and words (specifically trie based tokenizer) for script conversion (Pranav A et al., 2019).

Language Model Disambiguation: A strong baseline to this problem would be to build a language model to disambiguate between the characters, which is quite similar to STCP (Xu et al., 2017). We use a 2-layer LSTM language model trained on Traditional Chinese corpus.

Neural Sequence Models: We heuristically convert Traditional Chinese Wikipedia to Simplified Chinese using OpenCC and use it for training the seq2seq model (Sutskever et al., 2014). We

\footnotetext{
${ }^{5}$ If in case you are looking for 'Related Work' section.

${ }^{6}$ https://github.com/berniey/hanziconv

${ }^{7}$ https://github.com/hermanschaaf/mafan

${ }^{8}$ https://github.com/BYVoid/OpenCC
} 


\begin{tabular}{|c|c|c|c|c|c|c|c|c|c|c|}
\hline \multirow{2}{*}{ Conversion System } & \multicolumn{2}{|c|}{ HK Lit } & \multicolumn{2}{|c|}{ HK News } & \multicolumn{2}{|c|}{ TW Lit } & \multicolumn{2}{|c|}{ TW News } & \multicolumn{2}{|c|}{ Overall } \\
\hline & DED & SA & DED & SA & DED & SA & DED & SA & DED & SA \\
\hline Dictionary based conversion, Hanziconv & 34.1 & 54.7 & 37.7 & 59.1 & 31.3 & 60.0 & 39.3 & 58.9 & 34.2 & 55.6 \\
\hline Diction & 14.7 & 71.2 & 17.7 & 72.5 & 14.5 & 73.8 & 13.3 & 72.7 & 14.4 & 72.6 \\
\hline & 5.5 & 87.3 & 5.1 & 83.4 & 4.1 & 84.7 & 3.8 & 88.5 & 4.3 & 85.3 \\
\hline Langu & 6.3 & 85.6 & 5.4 & 79.9 & 4.7 & 84.1 & 5.2 & 83.9 & 5.3 & 84.0 \\
\hline Convolutior & 6.7 & 85.8 & 5.3 & 79.3 & 4.8 & 84.5 & 5.2 & 83.9 & 5.4 & 84.4 \\
\hline 2kenize wit & 11.2 & 84.3 & 12.1 & 81.3 & 11.3 & 82.1 & 10.0 & 81.1 & 11.5 & 82.7 \\
\hline 2kenize & 5.2 & 88.7 & $\underline{3.3}$ & 93.1 & $\underline{4.0}$ & $\underline{88.6}$ & 4.8 & 87.7 & 4.5 & 88.9 \\
\hline 2kenize with Unigram subwords & 3.4 & 91.9 & $\overline{3.8}$ & $\overline{90.9}$ & $\overline{4.3}$ & $\overline{88.1}$ & 3.9 & 87.8 & 3.7 & 89.3 \\
\hline 2kenize with joint LSTM modelling & $\overline{2.8}$ & $\overline{94.9}$ & 3.1 & 93.7 & 3.8 & 91.3 & $\overline{2.9}$ & $\overline{91.9}$ & $\overline{3.0}$ & $\overline{92.4}$ \\
\hline
\end{tabular}

Table 3: Results of the intrinsic evaluation experiments which are reported as a mean across 10 different seeds. We use disambiguation error density (DED, the lower, the better) and sentence accuracy (SA, the higher the better) metrics for evaluation. Bold: best, Underlined: second-best.

construct a 20-layer neural convolutional sequence model (Gehring et al., 2017) (both in encoder and decoder) using fairseq (Ott et al., 2019).

We perform ablation tests by inserting following segmentation models.

Word tokenization: We use Jieba, which is a commonly used hidden markov model based word tokenizer for Chinese NLP. ${ }^{9}$

Dictionary substrings: We apply maximal string matching, which is a dictionary based greedy tokenizer (Pranav A et al., 2019; Wong and Chan, 1996).

Unigram from Sentencepiece: Subword segmentation is performed by sampling unigram language model perplexity values (Kudo, 2018).

Joint subwords: As discussed in $§ 2.3$, we use joint SC-TC subwords.

\subsection{Results for Intrinsic Evaluation}

We evaluate our models using the metrics of disambiguation error density (DED) and sentence accuracy (SA). DED is the average of total edit distances per 1000 ambiguous Simplified characters, which is $\frac{\sum \text { edit distances }}{\sum \text { ambiguous Simplified characters }} \times 1000$. SA is the number of sentences correctly converted in percentages. Contrary to previous papers, we do not report character based accuracy values, as generally most characters have straightforward mappings - a reason why we opt for a less forgiving metric like SA where every character in a sentence has to be correctly converted.

Results are shown in Table 3, broken down by domain, and overall. Our model attains an average DED of 3.0 and a SA of $92.4 \%$ overall, whereas the best existing converter, OpenCC, only achieves a DED of 4.3 and a SA of $85.3 \%$. We

\footnotetext{
${ }^{9}$ https://github.com/fxsjy/jieba
}

find that seq2seq and LM based disambiguation perform almost on par with OpenCC, due to the large number of false positive errors by these models. Jieba achieves an average DED of 11.2 as it does not handle OOV words well. For maximal matching of segmented words and Unigram subwords, it achieves an overall DED of 4.5 and 3.7 , respectively - showing that joint segmentation yields better results. We observe that accuracy values are slightly worse on news text, due to the relatively high number of new entities in those datasets. We find that seq2seq and LM based disambiguation gives rise to many false positives. Heuristically converting TC to SC results in certain conversion errors in the training dataset; and additionally, seq2seq approaches tend to reword the target sentence, which shows that they are unsuitable for this task.

\subsection{Qualitative Error Analysis}

We manually inspect incorrect conversions in the intrinsic evaluation and find four interesting recurring linguistic patterns which confused the converters. We instructed the annotators to classify the items in the dataset (overall 12000 sentences in intrinsic evaluation dataset) if the sentences contain any of these patterns. In Table 4, we provide an overview of statistical information of these patterns and the performance by the converters.

Code mixing: Vernacular Cantonese characters (zh-yue) are a subset of TC characters but do not follow the norms of the standard written Chinese (Snow, 2004). We find that some of the sentences in our dataset are code-mixed with $z h$ yue (e.g. speech transcription) or English (e.g. named entities). Consider the snippet, “... 古惑架 BENZ 190E 撞埋支...”, which is code-mixed with 


\begin{tabular}{|c|c|c|c|c|}
\hline Case & Method & SA & Example & \\
\hline \multirow{5}{*}{$\begin{array}{l}\text { Code mixing } \\
\text { with } \\
\text { Cantonese } \\
(34 \text { cases, } \\
0.3 \%)\end{array}$} & & & 肯尼迪咁多嘢做, 掂唔掂呀? & $S C$ \\
\hline & & & With so much to do in Kennedy, can you handle it? & HK Lit \\
\hline & OpenCC & 20.5 & 肯尼迪咁多嘢做, 掂唔掂呀? & $x$ \\
\hline & STCP & 8.8 & 肯尼迪咁多嘢做, 掂唔掂呀? & $x$ \\
\hline & 2 kenize & 91.1 & 甘逎迪咁多嘢做, 掂唔掂呀? & $\checkmark$ \\
\hline \multirow{4}{*}{$\begin{array}{l}\text { Code mixing } \\
\text { with English } \\
\text { (1532 cases, } \\
12.8 \%)\end{array}$} & & & 自从我揸住大古惑架 BENZ 190E 撞埋支电灯柱㧽度之后, & $S C$ \\
\hline & & 95.6 & $\begin{array}{l}\text { After I drove Slick's Benz 190E into the telephone pole, } \\
\text { 自從我揸住大古惑架 BENZ 190E 撞埋支電燈柱嗰度之後, }\end{array}$ & HK Lit \\
\hline & STCP & 86.5 & 自從我揸住大古惑架 BENZ 190E 撞埋支電燈柱㧽度之后, & $x$ \\
\hline & 2kenize & 98.7 & 自從我揸住大古惑架 BENZ 190E 撞埋支電燈柱㧽度之後, & $\checkmark$ \\
\hline \multirow{5}{*}{$\begin{array}{l}\text { Disguised } \\
\text { Named } \\
\text { Entities } \\
(378 \text { cases, } \\
3.15 \%)\end{array}$} & & & 维护发展中国家共同利益 & $S C$ \\
\hline & & & Safeguard the common interests of developing countries & TW News \\
\hline & OpenCC & 85.7 & 維護髮展中國家共同利益 ～～～～～～～～～～ & $x$ \\
\hline & STCP & 82.1 & 維護髮展中國家共同利益 & $x$ \\
\hline & 2 kenize & 93.2 & 維護發展中國家共同利益 & $\checkmark$ \\
\hline Repeated & & & 乔治亚来到了乔治亚洲旅游 & $S C$ \\
\hline Named & & & Georgia came to Georgia for travelling. & HK News \\
\hline Entities & OpenCC & 84.4 & 佐治亞來到了佐治亞洲旅遊 & $x x$ \\
\hline (428 cases, & STCP & 17.9 & 佐治亞來到了喬治亞洲旅遊 & $x \checkmark$ \\
\hline $3.57 \%)$ & 2kenize & 87.8 & 喬治亞來到了喬治亞洲旅遊 & $\checkmark \checkmark$ \\
\hline
\end{tabular}

Table 4: Casewise breakdown of common errors. The first sentence is SC, second is the English translation and rest are TC outputs from the converters.

both zh-yue and English. The characters "BENZ $190 \mathrm{E}$ ”, 架 and 埋支 are not a part of standard written Chinese. We find that OOV words are 2 kenized into single-character tokens which results in: “古 惑 架 $|\mathrm{B}| \mathrm{E}|\mathrm{N}| \mathrm{Z} \mid$ 1|9|0|E| 撞 |埋|支” Thus, 2kenize distributes the entropy over multiple tokens rather than a single token (generally UNK is used in such cases). This allows the language model to have more space for multiple guesses, which shows a massive advantage over word models or just UNKing it, a reason why subword tokenizers outperform closed-vocabulary models (Merity, 2019).

Disguised Named Entities: Take the recurring sentence: “维护发展中国家共同利益”. Observe that the sentence contains a frequent word 中国 (China). However, the actual meaning and English translation do not include "China" at all. This is an interesting linguistic trait of Chinese, where words often appear in the sentence, but are not being interpreted. This could easily trip up a tokenizer, as the probability of 中国 being a token independently is high. Having 中国 as a separate token in the sentence could lead into an incorrect conversion (Table 1). We find in 2kenizer's trellis ${ }^{10}$ that “维 护|发展 |中” has a higher probability than other possible segmentations. Substructure lookups and beam search in our setup considerably reduces the probability of getting wrong tokenization. The sen-

\footnotetext{
${ }^{10}$ This is a probability lookup table in Viterbi to keep track of the segment information in a subsequence.
}

tence is 2 kenized into “维护 | 发展 | 中 | 国家 | 共 同 | 利益”, which results in the correct conversion - 維護發展中國家共同利益.

Repetitions: We find that in $3.57 \%$ of sentences, named entities are repeated. Interestingly, STCP, which uses a language model for disambiguation, often only converts one out of the repeated tokens correctly, which we can see in the table. As also shown, STCP prefers 佐治亞 over 喬治亞 in the first occurrence, but then prefers 喬治亞 ${ }^{11}$ in the second occurrence as it gets more context. 2kenize converts both of the entities correctly, very likely due to substructure lookups.

Failure Cases: Dictionary-based converters (OpenCC, HanziConv and Mafan) only use the first conversion candidate ${ }^{12}$ if multiple candidates are available. STCP often converts named entities wrongly, especially the ones which have longrange dependencies and repetitions. Although we find that 2kenize converts some of the unseen named entities perfectly, some of the errors caused were due to infrequent characters. Few cases are mainly related to variant characters ${ }^{13}$ which are often used interchangeably.

\footnotetext{
${ }^{11}$ Annotators and sources preferred 喬治亞 over 佐治亞.

${ }^{12}$ We are not sure how did they define the "order", but we have observed that they select more frequent characters as their most highly ranked ones.

${ }^{13}$ For example, 了解 and 瞭解 could be both used for "understand", and 裡面 and 裏面 could be used for “inside".
} 


\begin{tabular}{lr}
\hline \multicolumn{2}{c}{ Formal Text Classification Dataset Overview } \\
\hline Source & Singtao \\
Pretraining Corpus Size & 17500 \\
Training Size & 3000 \\
Validation Size & 450 \\
Testing Size & 450 \\
Categories & Financial, Educational, Local \\
& International, Sports \\
Language & zh-hant-hk \\
\hline \multicolumn{1}{c}{ Informal Text Classification Dataset Overview } \\
\hline Source & LIHKG \\
Pretraining Corpus Size & 21000 \\
Training Size & 4000 \\
Validation Size & 450 \\
Testing Size & 450 \\
Categories & Sports, Opinions, IT \\
& zh-hant-hk, zh-yue, en-HK \\
Languages &
\end{tabular}

Table 5: Characteristics of classification dataset (Traditional Chinese) for extrinsic evaluation experiments.

\section{Extrinsic Evaluation}

An accurate script converter should produce a less erroneous dataset, which should in turn improves the accuracy of the downstream tasks. In this section, we demonstrate the effect of script conversion on topic classification tasks to examine this assumption. We also study the impact of tokenization and pooling on the accuracy of topic classification. We apply the converter to the language modelling corpus (Wikitext), then train a classifier for informal and formal topic classification on that translated data. This allows us to measure the performance of the converter compared to other ones for a specific downstream task.

\subsection{Dataset for Extrinsic Evaluation}

This section describes the dataset that we used for extrinsic evaluation experiments. It involves a pretraining dataset which consists Chinese Wikipedia and topic classification datasets.

\subsubsection{Pretraining Dataset}

We use Chinese Wikipedia articles for pretraining the language model. Script conversion is an issue in Chinese Wikipedia, and currently, they use a server-side mechanism to automatically convert the scripts (dictionary-based) based on the location of the user. However, Wikipedia provides an option to view the article without conversion, which we use in the corpus. ${ }^{14}$ We use $z h-C N, z h-H K$ and $z h$-yue wikis to retrieve articles originally written $\mathrm{SC}, \mathrm{TC}$ and vernacular Cantonese $+\mathrm{TC}$ respectively with the help of wikiextractor. ${ }^{15}$ We pretrain the formal text classification models on articles from $z h-H K$ and converted $z h-C N$; and classification models for informal text on articles from $z h-H K, z h-y u e$, and converted $z h-C N$.

\subsubsection{Classification Datasets:}

We choose two classification tasks: formal news and informal topic classification (Table 5). For formal news, we scrape recent articles (2017-2019) from Singtao, ${ }^{16}$ for informal topics, we scrape posts (2017-2018) from LIHKG. ${ }^{17}$

\subsection{Performance of various classifiers}

For classification baselines, we use characterbased SVM (Support Vector Machines, Joachims (1998)), CNN (Convolutional Nets, Zhang et al. (2015)) and Chinese BERT (Devlin et al., 2019). We also employ a state-of-the-art text classifier, MultiFiT (Eisenschlos et al., 2019), a lightweight RNN-based language model based classifier, which has shown to achieve a performance competitive with BERT (Devlin et al., 2019) and ULMFiT (Howard and Ruder, 2018). The base architecture of MultiFiT is a 4-layer QRNN (Bradbury et al., 2016) with classifier head. We choose rectified Adam (Liu et al., 2019) with Lookahead (Zhang et al., 2019) as the optimizer. We employ the cosine cyclic learning scheduler (Smith, 2015), where the limits of learning rate cycles are found by increasing the learning rate logarithmically and computing the evaluation loss for each learning rate (Smith, 2018). To compute the batch size, we apply gradient noise scale to each batch size candidate and pick the one which gives the highest gradient noise scale (McCandlish et al., 2018). We apply label smoothing (Szegedy et al., 2015) and use mixed precision training on RTX 2080. We implement our experiments using Pytorch (Paszke et al., 2019) and FastAI (Howard and Gugger, 2020).

MultiFiT uses concat pooling after the last layer of QRNN, which means that the last time step is concatenated with an average and maximum

\footnotetext{
${ }^{14}$ Note that it would not be straight-forward to compare against Wikipedia's conversions directly, because they also perform some degree of manual post-processing (Contributors, 2019).

${ }^{15}$ https://github.com/attardi/wikiextractor

${ }^{16}$ https://std.stheadline.com

${ }^{17}$ https://lihkg. com
} 


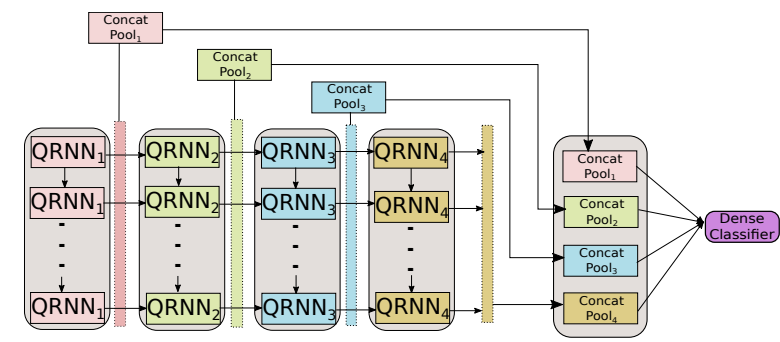

Figure 3: Proposed architecture for topic classification where we tweak MultiFiT to concatenate concat-pools from all layers. (Alt text: $§$ F.3).

\begin{tabular}{lrr} 
& Formal & Informal \\
\hline Char-SVM & 73.2 & 63.7 \\
Char-CNN & 78.5 & 64.9 \\
Chinese BERT (base) & 84.5 & 66.3 \\
MultiFiT with no pooling & 87.5 & 68.5 \\
MultiFiT with concat pooling & $\underline{88.6}$ & $\underline{69.9}$ \\
MultiFiT with layer pooling & $\mathbf{8 9 . 0}$ & $\underline{\mathbf{7 0 . 3}}$ \\
\hline
\end{tabular}

Table 6: Performance of various architectures on topic classification in terms of accuracy. The results are reported as a mean result across 10 different seeds and data splits. Bold: best, underlined: second best.

pooled over previous time steps. Studies show that in LM based classifiers, different layers capture different types of knowledge-the last layer would be domain-specific and initial layers would be more generalized (Yosinski et al., 2014; Peters et al., 2019). We speculate that concat pooling only on the last layer limits the information available to the classifier head and we hypothesise that the classifer would perform better if domainspecific as well as generalized knowledge were available to the head. For this reason, we augment the original MultiFIT architecture with layer pooling, which is concat pooling from all the layers, and pass that to the dense layer in the classifier, as shown in Fig 3.

We fine-tune the BERT language model and pretrain the MultiFiT language model on Chinese Wikipedia subsets (\$4.1.1). All classifiers are then trained on the given training set (character based models) and evaluated on the test set in terms of accuracy as number of items in each class are roughly equal. This experiment (and subsequent experiments in this section) is repeated across ten different seeds (Reimers and Gurevych, 2018) and data splits (Gorman and Bedrick, 2019) and the results are shown in Table 6. Layer pooling shows an absolute improvement of $0.4 \%$ improvement over concat pooling on formal and informal topic clas-

\begin{tabular}{lrr} 
Pretraining data of MultiFiT & Formal & Informal \\
\hline No Conversions & 89.0 & 70.3 \\
Including conversions with OpenCC & 91.7 & $\underline{75.6}$ \\
Including conversions with STCP & $\underline{92.3}$ & 73.4 \\
Including conversions with 2kenize & $\overline{\mathbf{9 3 . 2}}$ & $\mathbf{7 7 . 9}$ \\
\hline
\end{tabular}

Table 7: Ablation test of MultiFiT on different script converters. The results are reported as a mean accuracy result across 10 different seeds and data splits. Bold: best, underlined: second best.

\begin{tabular}{lrr} 
Corpus Tokenization & Formal & Informal \\
\hline Char & 93.2 & 77.9 \\
Jieba & 92.4 & 78.3 \\
BPE & 92.7 & 81.0 \\
BPE-Drop & $\underline{93.7}$ & $\underline{82.7}$ \\
Unigram & $\overline{\mathbf{9 4 . 8}}$ & 82.2 \\
1kenize & $\mathbf{9 4 . 8}$ & $\mathbf{8 3 . 2}$ \\
\hline
\end{tabular}

Table 8: Ablation test of MultiFiT on tokenizers. The results are reported as a mean accuracy result across 10 different seeds and data splits. Bold: best, underlined: second best.

sification, thus confirming our hypothesis.

\subsection{Effect of Conversion on Classification}

For each converter (OpenCC, STCP, 2kenize), we translate $z h-C N$ wiki dataset and augment it with the TC wiki dataset. Then, we pretrain on this dataset, finetune on the domain data and train MultiFiT with layer pooling on these three datasets. We demonstate test set accuracies in Table 7. The dataset translated by 2 kenize outperforms other converters, giving an absolute improvement of 0.9 $\%$ on formal and $4.5 \%$ over second-best converters on informal topic classification. These results emphasise that better script conversion improves the quality of the pretraining dataset, which boosts the performance of the downstream tasks like topic classification.

\subsection{Effect of Tokenization on Classification}

Studies show that tokenization affects classification accuracy; open-vocabulary methods generally perform best (Eisenschlos et al., 2019; Hiraoka et al., 2019). For this experiment, we perform further ablations on our previous best classifier setup (MultiFiT with layer pooling on 2 kenize) to understand the effect of various subword tokenizers. Pretraining generally takes a long time (1-2 GPU days), hence we pretrain the classifier once for each tokenized corpus and do not perform sub- 


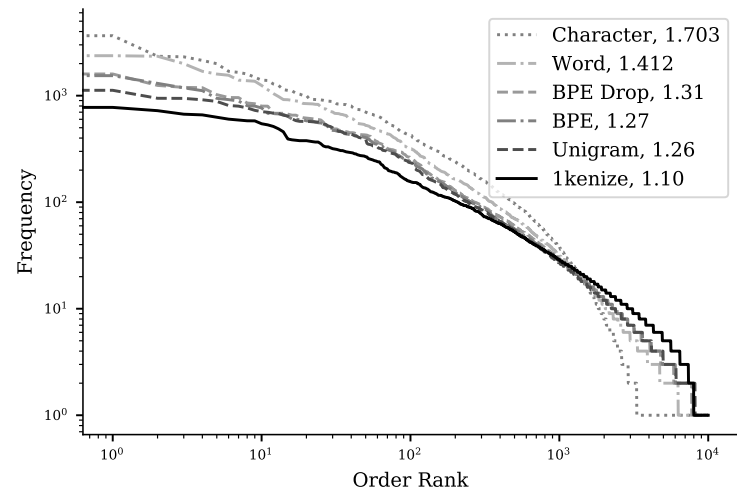

Figure 4: Log-log plots for different tokenizers. This is plotted frequency vs rank for the first 10000 tokens. Negative slopes calcuated from least squares are in the legend (lower means less skewed). (Alt text: §F.4).

word sampling for this experiment. For closed vocabulary methods, we use character and word segmentations (here with Jieba). Likewise, for openvocabulary methods, we employ BPE, BPE-Drop and Unigram subword tokenizers.

Subword tokenizers mostly rely on frequency and do not take likelihood (something similar to $n$-gram language model) of tokenized sentence into consideration. Hence, we choose LMbased Viterbi segmentation (henceforth referred as 1kenize), and here the LM would be the TC LSTM described in $§ 2.2$. We report results in Table 8. We find that for formal classification, 1 kenize and Unigram perform best. 1 kenize outperforms other subword tokenizers for the noisier informal dataset, giving an absolute improvement of $0.5 \%$ over the second best method, which is BPE-Drop.

We plot a log frequency of tokens vs log order rank, which is shown in Figure 4. This distribution is based on the LIHKG dataset, which is noisier than other domains. We observe that character and word distributions are steeper than language model based subword tokenizers. This indicates that subword tokenizers produce a less skewed token distribution. Subword tokenizers like BPE and Unigram are deterministic and rely on frequency for segmentation. Since 1 kenize is contextual, being LM-based, we find that it produces the least skewed distribution (lowest Zipf's law coefficient (Zipf, 1949)), which also reduces variance, a reason why this simple segmentation method outperforms others for informal text classification.

\section{Takeaways and Open Questions}

The contributions of our work are:
- 2kenize, a subword segmentation model, which jointly segments source sentence and its corresponding approximate target conversions.

- An unsupervised script converter based on 2 kenize which shows a significant improvement over existing script converters and supervised models.

- 1kenize, a variant of 2 kenize which performs tokenization on only Traditional Chinese sentences which improves accuracy on topic classification tasks.

- Character conversion evaluation datasets: spanning Hong Kong and Taiwanese literature and news genres.

- Traditional Chinese topic Classification datasets: formal (scraped from Singtao) and informal (scraped from LIHKG) styles spanning genres like news, social media discussions, and memes.

The key findings of our work are:

- Our script converter shows a strong performance when dealing with code mixing and named entities. Supervised models are prone to anaphora and unseen entities related errors.

- A simple LM-based Viterbi segmentation model outperforms other subword tokenizers on topic classification tasks and reduces skewness of token distribution on a noisy dataset.

We leave some open questions to explore:

- How can we exploit subword variations to reduce skewness in the NLU tasks?

- Would subword-segmentation-transfer be helpful for other NMT-NLU task pairs like we did for 2 kenize (script conversion) to lkenize (classification)?

We anticipate that this study would be useful to TC NLP practitioners, as we address several research gaps, namely script conversion and a lack of benchmark datasets.

\section{Acknowledgements}

The first author would like to thank Dayta AI Limited, S.F. Hui, I-Tsun Cheng, Ishaan Batra, Conrad Ho, Roy Fork, Abhishek Gupta, Ajay Singh, Eugene Ho, Patrick Tu, Alex Chu, and Leland So for making valuable additions to this work. The second author would like to acknowledge funding from the Swedish Research Council for the project under grant agreement 2019-04129, which partly funded this work. 


\section{References}

Emily M. Bender and Batya Friedman. 2018. Data statements for natural language processing: Toward mitigating system bias and enabling better science. Transactions of the Association for Computational Linguistics, 6:587-604.

James Bradbury, Stephen Merity, Caiming Xiong, and Richard Socher. 2016. Quasi-recurrent neural networks.

Chenhui Chu, Toshiaki Nakazawa, and Sadao Kurohashi. 2012. Chinese characters mapping table of Japanese, traditional Chinese and simplified Chinese. In Proceedings of the Eighth International Conference on Language Resources and Evaluation (LREC-2012), pages 2149-2152, Istanbul, Turkey. European Languages Resources Association (ELRA).

Wikipedia Contributors. 2019. Chinese script conversion and word processing in wikipedia. Page Version ID: 56925003.

Ryan Cotterell, Sabrina J. Mielke, Jason Eisner, and Brian Roark. 2018. Are all languages equally hard to language-model? In Proceedings of the 2018 Conference of the North American Chapter of the Association for Computational Linguistics: Human Language Technologies, Volume 2 (Short Papers), pages 536-541, New Orleans, Louisiana. Association for Computational Linguistics.

Piotr Czapla, Jeremy Howard, and Marcin Kardas. 2018. Universal language model fine-tuning with subword tokenization for polish. ArXiv, abs/1810.10222.

Paul Denisowski. 2019. Cc-cedict. https:// cc-cedict.org/.

Michael Denkowski and Graham Neubig. 2017. Stronger baselines for trustable results in neural machine translation. In Proceedings of the First Workshop on Neural Machine Translation, pages 18-27, Vancouver. Association for Computational Linguistics.

Jacob Devlin, Ming-Wei Chang, Kenton Lee, and Kristina Toutanova. 2019. BERT: Pre-training of deep bidirectional transformers for language understanding. In Proceedings of the 2019 Conference of the North American Chapter of the Association for Computational Linguistics: Human Language Technologies, Volume 1 (Long and Short Papers), pages 4171-4186, Minneapolis, Minnesota. Association for Computational Linguistics.

Julian Eisenschlos, Sebastian Ruder, Piotr Czapla, Marcin Kadras, Sylvain Gugger, and Jeremy Howard. 2019. MultiFiT: Efficient multi-lingual language model fine-tuning. In Proceedings of the 2019 Conference on Empirical Methods in Natural Language Processing and the 9th International Joint Conference on Natural Language Processing
(EMNLP-IJCNLP), pages 5706-5711, Hong Kong, China. Association for Computational Linguistics.

Thomas Emerson. 2005. The second international Chinese word segmentation bakeoff. In Proceedings of the Fourth SIGHAN Workshop on Chinese Language Processing.

A Foon. 1988. Diary of the little man. Book. Pp.5-6.

Jonas Gehring, Michael Auli, David Grangier, Denis Yarats, and Yann N. Dauphin. 2017. Convolutional sequence to sequence learning. In Proceedings of the 34th International Conference on Machine Learning - Volume 70, ICML'17, page 1243-1252. JMLR.org.

Kyle Gorman and Steven Bedrick. 2019. We need to talk about standard splits. In Proceedings of the 57th Annual Meeting of the Association for Computational Linguistics, pages 2786-2791, Florence, Italy. Association for Computational Linguistics.

Edouard Grave, Armand Joulin, and Nicolas Usunier. 2016. Improving neural language models with a continuous cache. ArXiv, abs/1612.04426.

Jack Halpern and Jouni Kerman. 1999. Pitfalls and complexities of chinese to chinese conversion. In International Unicode Conference (14th) in Boston.

Tatsuya Hiraoka, Hiroyuki Shindo, and Yuji Matsumoto. 2019. Stochastic tokenization with a language model for neural text classification. In Proceedings of the 57th Annual Meeting of the Association for Computational Linguistics, pages 16201629 , Florence, Italy. Association for Computational Linguistics.

Elad Hoffer, Itay Hubara, and Daniel Soudry. 2017. Train longer, generalize better: closing the generalization gap in large batch training of neural networks. In NIPS.

Jeremy Howard and Sylvain Gugger. 2020. Fastai: A layered api for deep learning. Information, 11(2):108.

Jeremy Howard and Sebastian Ruder. 2018. Universal language model fine-tuning for text classification. In Proceedings of the 56th Annual Meeting of the Association for Computational Linguistics (Volume 1: Long Papers), pages 328-339, Melbourne, Australia. Association for Computational Linguistics.

Jiubadao. 2011. You Are the Apple of My Eye. Chun Tian Chu Ban.

Thorsten Joachims. 1998. Text categorization with support vector machines: Learning with many relevant features. In $E C M L$.

Kazuya Kawakami, Chris Dyer, and Phil Blunsom. 2017. Learning to create and reuse words in openvocabulary neural language modeling. In Proceedings of the 55th Annual Meeting of the Association for Computational Linguistics (Volume 1: Long Papers), pages 1492-1502, Vancouver, Canada. Association for Computational Linguistics. 
Urvashi Khandelwal, He He, Peng Qi, and Dan Jurafsky. 2018. Sharp nearby, fuzzy far away: How neural language models use context. In Proceedings of the 56th Annual Meeting of the Association for Computational Linguistics (Volume 1: Long Papers), pages 284-294, Melbourne, Australia. Association for Computational Linguistics.

Yoon Kim, Alexander Rush, Lei Yu, Adhiguna Kuncoro, Chris Dyer, and Gábor Melis. 2019. Unsupervised recurrent neural network grammars. In Proceedings of the 2019 Conference of the North American Chapter of the Association for Computational Linguistics: Human Language Technologies, Volume 1 (Long and Short Papers), pages 11051117, Minneapolis, Minnesota. Association for Computational Linguistics.

Giddens Ko. 2010. Cafe, Waiting, Love. Spring Press.

Taku Kudo. 2018. Subword regularization: Improving neural network translation models with multiple subword candidates. In Proceedings of the 56th Annual Meeting of the Association for Computational Linguistics (Volume 1: Long Papers), pages 6675, Melbourne, Australia. Association for Computational Linguistics.

Cheung Lau Yee. 1972. Intersection. Benefits Publishing Co., Ltd.

Liyuan Liu, Haoming Jiang, Pengcheng He, Weizhu Chen, Xiaodong Liu, Jianfeng Gao, and Jiawei Han. 2019. On the variance of the adaptive learning rate and beyond. ArXiv, abs/1908.03265.

Yichang Liu. 1962. Drunkard. Benefits Publishing Co., Ltd.

Sam McCandlish, Jared Kaplan, Dario Amodei, and OpenAI Dota Team. 2018. An empirical model of large-batch training. ArXiv, abs/1812.06162.

Stephen Merity. 2019. Single headed attention rnn: Stop thinking with your head. ArXiv, abs/1911.11423.

Sabrina J. Mielke. 2019. Can you compare perplexity across different segmentations?

Sabrina J. Mielke, Ryan Cotterell, Kyle Gorman, Brian Roark, and Jason Eisner. 2019. What kind of language is hard to language-model? In Proceedings of the 57th Annual Meeting of the Association for Computational Linguistics, pages 4975-4989, Florence, Italy. Association for Computational Linguistics.

Sabrina J. Mielke and Jason Eisner. 2019. Spell once, summon anywhere: A two-level open-vocabulary language model. $A A A I$.

Tomáš Mikolov, Ilya Sutskever, Anoop Deoras, HaiSon Le, Stefan Kombrink, and Jan Cernocky. 2012. Subword language modeling with neural networks. preprint (http://www. fit. vutbr. cz/imikolov/rnnlm/char. pdf), 8.
Masaaki Nagata. 1994. A stochastic Japanese morphological analyzer using a forward-DP backward-A* n-best search algorithm. In COLING 1994 Volume 1: The 15th International Conference on Computational Linguistics.

Xue Nianwen, Zhang Xiuhong, Jiang Zixin, Palmer Martha, Xia Fei, Chiou Fu-Dong, and Meiyu Chang. 2016. Chinese treebank 9.0. LDC2016T13. Web Download. Philadelphia: Linguistic Data Consortium.

Myle Ott, Sergey Edunov, Alexei Baevski, Angela Fan, Sam Gross, Nathan Ng, David Grangier, and Michael Auli. 2019. fairseq: A fast, extensible toolkit for sequence modeling. In Proceedings of the 2019 Conference of the North American Chapter of the Association for Computational Linguistics (Demonstrations), pages 48-53, Minneapolis, Minnesota. Association for Computational Linguistics.

Adam Paszke, Sam Gross, Francisco Massa, Adam Lerer, James Bradbury, Gregory Chanan, Trevor Killeen, Zeming Lin, Natalia Gimelshein, Luca Antiga, Alban Desmaison, Andreas Dipl.-Ing. Kopf, Edward Yang, Zach DeVito, Martin Raison, Alykhan Tejani, Sasank Chilamkurthy, Benoit Steiner, Lu Fang, Junjie Bai, and Soumith Chintala. 2019. Pytorch: An imperative style, high-performance deep learning library. In NeurIPS 2019.

Matthew E. Peters, Sebastian Ruder, and Noah A. Smith. 2019. To tune or not to tune? adapting pretrained representations to diverse tasks. In Proceedings of the 4th Workshop on Representation Learning for NLP (RepL4NLP-2019), pages 7-14, Florence, Italy. Association for Computational Linguistics.

Pranav A, S.F. Hui, I-Tsun Cheng, Ishaan Batra, and Chiu Yik Hei. 2019. Learn languages first and then convert: Towards effective simplified to traditional chinese conversion. In Proceedings of the 2019 Conference of the North American Chapter of the Association for Computational Linguistics (Student Research Workshop, non-archival), Minneapolis, Minnesota. Association for Computational Linguistics.

Ivan Provilkov, Dmitrii Emelianenko, and Elena Voita. 2019. Bpe-dropout: Simple and effective subword regularization. ArXiv, abs/1910.13267.

Nils Reimers and Iryna Gurevych. 2018. Why comparing single performance scores does not allow to draw conclusions about machine learning approaches.

Rico Sennrich, Barry Haddow, and Alexandra Birch. 2016. Neural machine translation of rare words with subword units. In Proceedings of the 54th Annual Meeting of the Association for Computational Linguistics (Volume 1: Long Papers), pages 17151725, Berlin, Germany. Association for Computational Linguistics. 
Xiaodong Shi, Yidong Chen, and Xiuping Huang. 2011. Key problems in conversion from simplified to traditional chinesecharacters. In International Conference on Asian Language Processing.

Leslie N. Smith. 2015. Cyclical learning rates for training neural networks. 2017 IEEE Winter Conference on Applications of Computer Vision (WACV), pages 464-472.

Leslie N. Smith. 2018. A disciplined approach to neural network hyper-parameters: Part 1 - learning rate, batch size, momentum, and weight decay. ArXiv, abs/1803.09820.

Don Snow. 2004. Cantonese as written language: The growth of a written Chinese vernacular, volume 1. Hong Kong University Press.

Richard W. Sproat, Chilin Shih, William Gale, and Nancy Chang. 1996. A stochastic finite-state wordsegmentation algorithm for Chinese. Computational Linguistics, 22(3):377-404.

Martin Sundermeyer, Ralf Schlüter, and Hermann Ney. 2012. Lstm neural networks for language modeling. In INTERSPEECH.

Ilya Sutskever, Oriol Vinyals, and Quoc V Le. 2014. Sequence to sequence learning with neural networks. In Z. Ghahramani, M. Welling, C. Cortes, N. D. Lawrence, and K. Q. Weinberger, editors, Advances in Neural Information Processing Systems 27, pages 3104-3112. Curran Associates, Inc.

Christian Szegedy, Vincent Vanhoucke, Sergey Ioffe, Jon Shlens, and Zbigniew Wojna. 2015. Rethinking the inception architecture for computer vision. 2016 IEEE Conference on Computer Vision and Pattern Recognition (CVPR), pages 2818-2826.

Thomas Wolf, Lysandre Debut, Victor Sanh, Julien Chaumond, Clement Delangue, Anthony Moi, Pierric Cistac, Tim Rault, R'emi Louf, Morgan Funtowicz, and Jamie Brew. 2019. Huggingface's transformers: State-of-the-art natural language processing. ArXiv, abs/1910.03771.

Pak-kwong Wong and Chorkin Chan. 1996. Chinese word segmentation based on maximum matching and word binding force. In COLING 1996 Volume 1: The 16th International Conference on Computational Linguistics.

Jiarui Xu, Xuezhe Ma, Chen-Tse Tsai, and Eduard Hovy. 2017. Stcp: Simplified-traditional chinese conversion and proofreading. Proceedings of the IJCNLP 2017, System Demonstrations, pages 6164.

Chiung Yao. 1964. Fire and rain. Book. ISBN 0-33036076-0.

Jason Yosinski, Jeff Clune, Yoshua Bengio, and Hod Lipson. 2014. How transferable are features in deep neural networks? In NIPS.
Michael Ruogu Zhang, James Lucas, Geoffrey E. Hinton, and Jimmy Ba. 2019. Lookahead optimizer: k steps forward, 1 step back. ArXiv, abs/1907.08610.

Xiang Zhang, Junbo Jake Zhao, and Yann LeCun. 2015. Character-level convolutional networks for text classification. In NIPS.

Xiaoheng Zhang. 2014. A comparative study on simplified-traditional chinese translation. In Chinese Computational Linguistics and Natural Language Processing Based on Naturally Annotated Big Data, pages 212-222. Springer.

George Kingsley Zipf. 1949. Human behavior and the principle of least effort. 


\section{A Summary in Traditional Chinese: 簡 體中文到繁體中文的文本轉換器}

研究中文 NLP 時, 將文本進行繁簡轉換是常 見的數據預處理步驟。在簡繁轉換過程中, 經 常出現多個繁字轉換成同一簡體字, 反之亦 然。藉此透過測試現行的繁簡轉換算法, 發 現只有 55-85\% 隼確度。進一步的調查發現， 現代的神經網絡，譬如神經語言模型的字符 歧義消除 (neural language model character disambiguation) 和神經序列模型 (neural sequence models)，均只達到 84-85\% 的句子準確性，都 是由第一類錯誤 (Type I error) 所致。我們推 斷上述問題, 是由於模型未能有效嶅清子詞 (subword) 的邊界所導致。

在此, 我們提出了 2kenize, 一個子詞分割 模型 (subword segmentation model)，同時利用 先行式繁體中文以及簡體中文進行建構。我們 將聯合簡體中文及繁體中文共同訓練 Viterbi 分詞器。即使利用較具挑戰性的數據集測試, 本模型亦達到 $91-95 \%$ 消歧準確度。透過定 性誤差分析 (qualitative error analysis), 展示了 本模型更擅長處理 code-mixing 以及命名個體 (named entities) 除此以外，我們亦在主題分類 領域中進行了外部評估, 本模型更在主題分 類的字符及詞語模型 (character and word-based models) 的領域中表現出眾, 更在子詞正則化 (subword regularization) 中, 獲得比 BPE 更好 的名次。然後針對繁體中文句子對 2 kenize 進 行調整, 誕生了 1 kenize。1 kenize 分別在正式 數據集與其他子詞分詞器 (subword tokenizers) 名列前茅, 在非正式數據集上更表現超群。由 此, 我們推斷子詞分詞器會嚴重地受 token 的 分佈及偏度而影響

\section{是次研究的貢獻：}

1. 2 kenize : 簡體中文到繁體中文的文本轉 換器

2. 字符轉換評估數據集：跨越香港和台灣文 獻及新聞等多個類型的數據集

3. 主題分類數據集 : 繁體中文的正式和非正 式文本數據涵蓋新聞，社交媒體討論，改 圖，改歌，memes 等二次創作文本。

\section{B Data Statement for Intrinsic Evaluation}

\section{B.1 Corpus}

In this subsection, we discuss the annotation procedure and the characteristics of the corpus used for the intrinsic evaluation. We have used Bender and Friedman (2018) data statement design for the description.

\section{B.1.1 Curation Rationale}

The script conversion task is understudied in NLP and we could not find good quality parallel corpora to evaluate our approaches. The idea is to curate a diverse collection of TC works and convert them to $\mathrm{SC}$, due to its one-to-one correspondence. However, we find out that some of the conversions were wrong because

1. sometimes dictionaries resulted in incorrect conversion,

2. stylistic differences between HK and TW characters and phrasing,

3. code-mixing of Cantonese and Traditional Chinese,

4. code-mixing with non-Chinese characters,

5. some characters in TC-SC conversion have one-to-many mappings as well.

Hence, we need quality control with human annotators to validate our conversions.

\section{B.1.2 Annotation Process}

Demographic: We opted for 4 trained annotators, 2 for annotating HK-style TC and 2 for annotating TW-style TC and thus going for double annotation for the corpus. They ranged in age from $18-20$ years, included 2 men and 2 women, gave their ethnicity as Hong Kongers (2) and Taiwanese (2), and their native spoken languages were Cantonese (2) and Taiwanese Mandarin (2).

Workload: Annotators approximately validated 100 sentences per hour, comprising of total workload of 60 hours. They were given a month to annotate and were paid 5000 Hong Kong Dollars on completion.

Procedure: The annotators were shown TC and converted SC sentences (we used OpenCC to convert) and were asked to validate and correct any conversion mistakes. In case of disagreement, we used majority voting between automatically converted and annotators' corrections.

We provide raw agreement and Krippendorf' $\mathrm{s} \alpha$ in Table 1 for pooled data and various sub-groups of the dataset. We also report inter-annotator agreements on character and phrasal levels in Table 2. These agreement values are difficult to interpret, but generally $\alpha \geq 0.8$ is considered to be substantial. 


\begin{tabular}{ll|cc}
\hline & & RA & $\alpha$ \\
\hline HK & & 0.98 & 0.98 \\
& Lit & 0.982 & 0.98 \\
& News & 0.979 & 0.97 \\
\hline TW & & 0.98 & 0.98 \\
& Lit & 0.981 & 0.98 \\
& News & 0.971 & 0.97 \\
\hline
\end{tabular}

Table 9: Inter-annotator agreements

\begin{tabular}{lll} 
& RA & $\alpha$ \\
\hline Character Level & 0.98 & 0.97 \\
Word Level & 0.95 & 0.94 \\
Sentence Level & 0.93 & 0.92
\end{tabular}

Table 10: Inter-annotator agreements as per different levels

\section{B.1.3 Speech Situation}

The publication dates and sources are listed in the Table 2. HK and TW literature consists of popular books for which many movie and drama adaptations are made. ${ }^{18}$ Specifically, for HK literature, the text contains code-mixed characters with Vernacular Cantonese, which is quite unusual in formal publishing practices, and these books are often cited as an example for popularizing Cantonese in the 60s (Snow, 2004). We also found code-mixing with English and numerous transliterated named entities which we have used for qualitative error analysis in the Table 4.

\section{B.1.4 Text Characteristics}

Although Hong Kong and Taiwan both use Traditional Chinese, they are stylistically different as the dominant spoken language in $\mathrm{HK}$ is Cantonese and in TW is Taiwanese Mandarin. Thus, it is quite essential to test the performance of our algorithms on these two styles. We collected two genres for each style: informal literature and formal news. We found more variation within informal HK-TW literature as compared to the formal news. We intentionally chose long sentences (average length of 200 characters), especially which contain more ambiguous characters to make the dataset more challenging for testing.

\section{Data Statement for Extrinsic Evaluation}

This subsection describes the characteristics of the topic classification in Traditional Chinese. For the

\footnotetext{
${ }^{18}$ We highly recommend these movies and novels as well.
}

short overview, please see Table 5.

\section{C.1 Curation Rationale}

We choose two different styles for curating this dataset: formal and informal. The formal text consists of news dataset scraped from Singtao, one of the popular newswire in Hong Kong. The classes in this dataset consist of Financial, Educational, Local, International, and Sports subsections. There are 17500 unlabelled and 3900 labelled items in this section. Authors would like to credit I-Tsun Cheng for giving us helpful suggestions in curating this dataset.

The informal text consists of social media posts dataset scraped from LIHKG, a Twitter equivalent in Hong Kong. The classes in this dataset consist of Sports, Opinions, Memes, IT, Financial and Leisure. There are 21000 unlabelled and 4900 labelled items in this section. Authors would like to credit Leland So for giving us helpful suggestions in curating this dataset.

\section{C.2 Language Variety}

The texts in the formal subsection are typically written in Hong Kong style Traditional Chinese (zh-hant-hk). The posts scraped from LIHKG are predominantly in Traditional Chinese (zh-hant$\mathrm{hk}$ ), and they are often code-mixed with Vernacular Cantonese (zh-yue) and English (en-HK).

\section{C.3 Speaker Demographic}

Speakers were not directly approached for inclusion in this dataset and thus could not be asked for demographic information. Our best guess for demographic of LIHKG forum users are typically university students (19-23 years), and the majority of them speak Cantonese as a native language.

\section{C.4 Text Characteristics}

The news articles are scraped from 2017-2019 and LIHKG posts are scraped from 2017-2018. Some of the posts in LIHKG are in the transliterated Cantonese form and some of them are not written in Standard Written Chinese. The news posts are generally quite long and often contains more than 5 sentences (average length of nearly 300 characters). On the other hand, the LIHKG posts are shorter and forums titles are generally one sentence each (average length of nearly 50 characters). Please note that due to the current situations in Hong Kong, we do not include political posts and news from mid-2019. 


\section{Description of Intrinsic Evaluation Experiments}

\section{D.1 Heuristic Grid Search of Learning Rate and Batch Size Hyperparameters}

We employ the cosine cyclic learning scheduler (Smith, 2015), where the limits of learning rate cycles are found by increasing the learning rate logarithmically and computing the evaluation loss for each learning rate (Smith, 2018). To compute the batch size, we apply gradient noise scale to each batch size candidate and pick the one which gives the highest gradient noise scale (McCandlish et al., 2018).

\section{D.2 Training of SC and TC Language Model}

The datasets are described in $\S 3.2$. The model architecture is 2-layer LSTM language model with tied weights. Embedding size is 512 and hidden size is 512 . We perform a concat pooling in the last layer where we concatenate the last output of the word, mean pool and max pool of all representations. We adopt comparable subword perplexity as suggested by Cotterell et al. (2018); Mielke et al. (2019); Mielke (2019), where we use a common denominator, referring to the number of segments per word in order to compare. On average, we achieve a perplexity of 168.6 on the Chinese Treebank test set (Nianwen et al., 2016). Also refer to Chinese LM Benchmark: https://chinesenlp.xyz/\#/docs/ language_modeling. The training took 2 days on RTX 2080 with FP16 training, with a batch size of 256 and number of epochs of 250.

\section{D.3 Training of Convolutional seq2seq}

Training dataset is a heuristically converted Traditional Chinese Wikipedia with OpenCC. We use 20 layers in encoder and decoder with the embedding size of 512 implemented in Fairseq (Ott et al., 2019). Dropout is 0.1 and we use adaptive softmax to speed up the training. The training took 1 day on RTX 2080 with FP16 training, with a batch size of 128 and number of epochs of 250.

\section{E Description of Extrinsic Evaluation Experiments}

\section{E.1 Character CNN training}

The datasets are described in $\$ 4.1 .2$. The model architecture is 7-layer CNN with tied weights and residual blocks. Embedding size is 512 and hidden size is 512 . We perform a concat pooling in the last layer where we concatenate the last output of the word, mean pool and max pool of all representations. The training took 16 hours on RTX 2080 with FP16 training, with a batch size of 256 and number of epochs of 350 .

\section{E.2 Chinese BERT training}

The datasets are described in \$4.1.2. We use Chinese BERT base (12-layer, 768-hidden, 12heads, $110 \mathrm{M}$ parameters) using Transformers library (Wolf et al., 2019). We use sequence length of 384 and batch size of 12 . Finetuning language model took 2 hours (learning rate of $3 e-5$ ) and finetuning classifier took 1 hour each on both datasets, including grid search on learning rates: $3 \mathrm{e}-4,1 \mathrm{e}-$ $4,5 e-5,3 e-5$, where $3 e-5$ gives the best results (on RTX 2080 with FP16 training).

\section{E.3 MultiFiT training}

We found MultiFiT is highly reproducible as compared to other models as it gives the least variance across the seeds and data splits. Hyperparameters are chosen by heuristic grid search on learning rate and batch size. The datasets are described in \$4.1.2. Pretraining language model takes $1 \mathrm{GPU}$ day for each experiment of MultiFiT. Finetuning language model takes 3 hours where we used a patience of 2 epochs. Finetuning classifiers takes 3 hours where we used a patience of 2 epochs. All experiments of MultiFiT are implemented using FastAI (Howard and Gugger, 2020).

\section{F Alternative texts for figures and Chinese explanations}

\section{F.1 Alternative text for Figure 1}

The recurring Chinese sentence is split and we take one subsequence of it. The other subsequence is used in next iteration. We perform Unigram viterbi segmentation on this and get the probabilities. The probabilities are normalized and we sample a segmentation using this probability. This segmentation goes into the model which goes through cached embeddings, followed by stacked LSTM layers, followed by concat pooling (which consists of last output, mean pooling and max pooling) which then goes through a linear layer. We cache the top-k embeddings in the main memory and for the least frequent embeddings we track the gradients and do not keep them in the main network (we used gradient accumulation). 


\section{F.2 Alternative text for Figure 2}

From the given SC sentence, we create possible TC sequences using mappings. We input these to Viterbi, which recursively calls LSTM. Using Eq. (6) as the scoring function, Viterbi outputs the mapping sequence. We perform beam search to find the best TC sequence from the mapping sequence where we used the same TC LSTM again.

\section{F.3 Alternative text for Figure 3}

The architecture contains 4 stacked QRNN layers. Each layer has QRNN cells. After every layer we perform a concat pool (taking the last output, max pool and mean pool). We aggregate these pools in the final layer which goes into a linear layer. We highly recommend this for making the training more stable.

\section{F.4 Alternative text for Figure 4}

We have plotted log-log token distribution. On xaxis we have order rank and on y-axis we have frequencies. Character based tokenization gives a slope of 1.703, BPE-Drop gives 1.31, BPE gives 1.27 , word tokenization (Jieba) gives 1.41 , unigram sampling gives 1.28 and 1 kenize gives the least skewed distribution with a slope of 1.1. Note that these are negative slope and lower the slope is, more efficiently vocabulary is tokenized.

\section{F.5 Recurring Chinese sentence}

Here, we explain the recurring sentence in this paper. In Table 1 we had SC sentence 维护发展中国 家共同利益, which means Safeguarding the common interests of developing countries. This is pronounced as Wéihù fāzhăn zhōng guójiā gòngtóng liỳi in Mandarin. Its correct TC translation is 維 護發展中國家共同利益, which is pronounced as wai4 wu6 faat 3 zin2 zung1 gwok3 gaal gung6 tung4 lei6 jik1 in Cantonese (note that the numerals are the tones). 